\title{
Evaluation of the Effects of Environmental Exposure on the Performance Decay of ETICS
}

\author{
Maurizio Nicolella ${ }^{1}$, Roberto Landolfi ${ }^{2}$ and Alessio Pino ${ }^{3}$ \\ ${ }^{1}$ University of Naples Federico II, Department of Civil, Architectural and Environmental \\ Engineering, Piazzale Vincenzo Tecchio, 80,80125 Naples, Italy, maurizio.nicolella@unina.it \\ ${ }^{2}$ University of Naples Federico II, Department of Civil, Architectural and Environmental \\ Engineering, Piazzale Vincenzo Tecchio, 80, 80125 Naples, Italy, roberto.landolfi@unina.it \\ ${ }^{3}$ University of Naples Federico II, Department of Civil, Architectural and Environmental \\ Engineering, Piazzale Vincenzo Tecchio, 80, 80125 Naples, Italy, alessio_pino@hotmail.it
}

\begin{abstract}
The performance decay of a building component is influenced by a huge variety of parameters, interacting between them and determining different results in terms of service life and performance over time. For this reason, evaluating and modeling the performance of a component requires the comprehension of the way and the measure the single factors affect it, and turning this into an analytical model is a very complex operation. Moreover, components of existing buildings are subjected to an additional incognita: the characteristics and the durability of the materials, which is hardly a certain information, and can vary between similar buildings, affecting the accuracy of the evaluation. This study, conducted according to the guidelines of ISO 15686:7 code, investigates on a common solution for interventions on existing buildings, the ETICS, surveying their current performance degree through thermographic camera tests. The aim is to appreciate the difference in the performance decay between different façades of the same building - a homogeneity that allows to estimate this parameter alone - in order to reach an analytical expression of the variation of performance decay according to the difference in the exposure. The sample is constituted by buildings of large dimensions and well-known original projects and details of successive interventions, located in the city of Naples. The data obtained with in-field measurements were subjected to statistical analysis, to allow the quantitative determination of the impact of this parameter.
\end{abstract}

Keywords: Durability, ETICS, in-field Measurement.

\section{Introduction and State of the Art}

In the last decades, together with the acknowledgement of the necessity to plan the maintenance of a building in advance, an increasingly higher importance has been given to the role of the comprehension of the dynamics of performance decay of building materials and components. In fact, knowing the trends and the times of performance decay is a required item, in order to predict the performance levels that will be reached through time, according to which maintenance interventions can be chosen.

Since diseconomies in terms of maintenance choices evidently lead to a waste of resources, the consequences of a scarce knowledge about the performance decay of components rapidly produce effects on the aspects of sustainability and energy consumption, which is a critical topic in the current situation of the construction industry. Despite that, accomplishing a high level of detail in the understanding of the processes that determine the performance decay of building components is definitely not an easy task. On one hand there are the intrinsic 
characteristics of the materials, which can also be determined through laboratory tests which simulate the passing of time (i.e. accelerated aging tests), but on the other hand the actual inuse condition of building components has several possible variations: the micro-context, and macro-context of the building, the compatibility with other components, and many more. This evidence is also embodied in the structure of the Factor Method, which attributes a separate weight for the external and internal environment.

It seemed interesting to refer to a specific component for which the environmental impact of the maintenance activity has been emphasized (Minarovičová, 2015), that is used to produce benefits in terms of durability of the system, and improvement of thermal - so, energy - performance: the ETICS or External Thermal Insulation Composite Systems.

They consist in an insulation board bonded onto a new or existing masonry or concrete wall, and mechanically fixed with anchors. The insulation board is then protected by a base coat, usually 3-4 mm thick, made of the cement mortar with resin used as adhesive, but reinforced with a glass fibre mesh. Onto the base coat a "key coat', or primer, is applied, and then the finishing, which is usually a thick $(1.5-2 \mathrm{~mm})$ top coat. This technology is very common all over Europe, thanks to its low cost and the possibility it offers to accomplish a very good improvement of the thermal properties of the building envelope.

The research investigates the variation of the trend of its performance decay according to the environmental exposure, and in particular the cardinal exposure: considering that very often it is applied on the whole surface of buildings, then the differences in the behavior of the ETICS on the single façades are an interesting item.

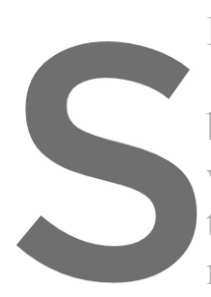

\section{This kind of solutio} buildings - Design and with renders (ETICS) the field of research. making use of climatic
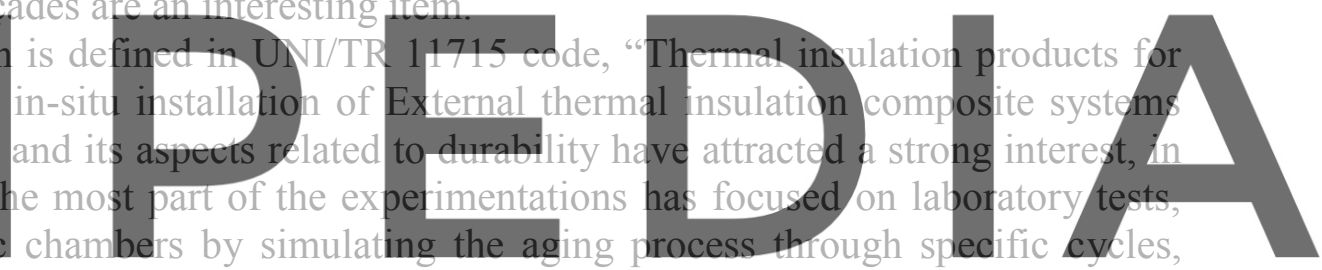

among which the first attempts were performed by Daniotti and Paolini (2005), who realized

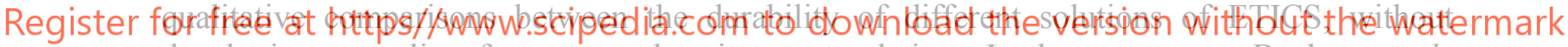
developing re-scaling factors to relate it to natural time. In the same year, Bochen et al., (2005) performed other climatic chamber tests, also determining the equivalence to natural aging. Years later, Griciuté et al., (2013) made more experimentations involving the accelerated ageing of ETICS.

Other researchers investigated specific aspects of the durability of ETICS, among which Topcu and Merkel (2008), who focused on the moisture absorption, Künzel and Zirkelbach (2008), who studied the influence of rain water leakage on the hygrothermal performance of ETICS. There has been, in general, a much higher number of laboratory experimentations than field tests, including computational and theoretical modelling of the durability of this solution, such as the computational analysis performed by Koči et al., (2012) and the multiphysics modelling by Daniotti et al., (2014). On the other hand, there has been a much lower, and more recent, number of field researches on the durability of ETICS, realized by De Freitas (2016) and Kvande (2018). In these and other cases, little use of the thermographic camera has been noticed. 


\section{Materials and Methods}

The investigated solution of ETICS has the following structure:

- skim "Klebocem", width $0.5 \mathrm{~cm}$;

- SK insulating panel "Stiferite", width $4 \mathrm{~cm}$;

- mechanical anchors in PVC, 2 per $\mathrm{m}^{2}$;

- primer "Klebocem";

- glass fiber mesh, width $0.3 \mathrm{~cm}$;

- primer "Klebocem";

- colored top coat "Rivaton", width 0.2-0.3 cm.

The research has been carried out as a thermographic and photographic analysis on ten buildings in Naples, Italy - in Via Attila Sallustro - which have been built in 2012-2013. They are all buildings in reinforced concrete, consisting of seven floors and a total lateral surface, constituted by hollow bricks and ETICS, of $25000 \mathrm{~m}^{2}$. The device used for the thermographic survey was a thermographic camera IR FLIR 250T, and the output consisted in a total of sixty thermographic pictures, taken from the ground level, which show several relative differences in the superficial temperature, highlighting phenomena of detachment which could not, in most of the cases, be noticed by simple visual inspection. The individuation of the performance level of the various parts of the building followed the procedure suggested in ISO 15686-7 code.

Also, in accordance with the recommendations from UNI EN ISO 4628-1 code, it is

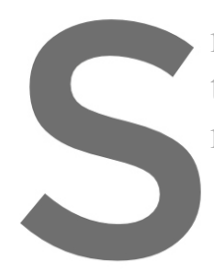

necessary to define performance

to attribute quantitative

represent the in-use con

Table 1. Performance degrees for
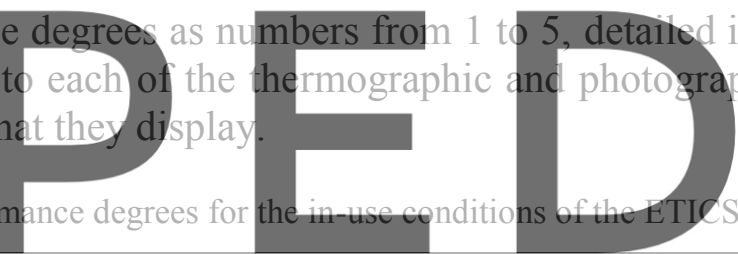

Degree

Type of symptoms

In-use condition of the ETICS

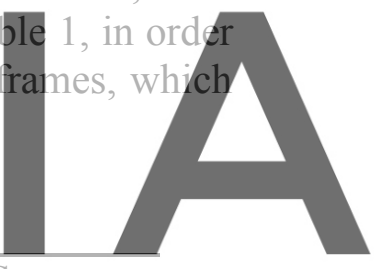

Register for free at https//www.scipedia.com to downloadperfermersionecaythout the watermark Slight symptoms

Cracking and blistering phenomena on the surface (millimetrical size)

\begin{tabular}{ccc}
3 & Medium symptoms & $\begin{array}{c}\text { Macro-cracking and macro-blistering } \\
\text { phenomena on the surface (centimetrical size) }\end{array}$ \\
\hline 4 & Strong symptoms & Slight detachment of the panels \\
\hline 5 & Totally unacceptable & Partial/total detachment of the panels \\
\hline
\end{tabular}

The definition of the performance degrees displays two typologies of anomalies: the "slight symptoms" and the "medium symptoms" refer to the finishing layers, which have a basically protective performance and effect on the panels, while the "strong symptoms" and the "totally unacceptable" state are related to the panels themselves, and therefore have a much stronger significance in terms of performance decay of the ETICS.

In several cases, the thermographic frames show thermal gradients - caused by detachments - also in locations where no performance decay is visible by sight and by photographic frames, nor is related to an anomaly in the superficial layers, as acknowledged through tactile contact: this means that their origin has to be linked to an anomaly in the inner layers, presumably the panels themselves. 
Figure 1 shows the distribution of the optical cones of the thermographic and photographic frames, numbered from 1 to 17 ; for each of them, 3-5 shots have been taken.

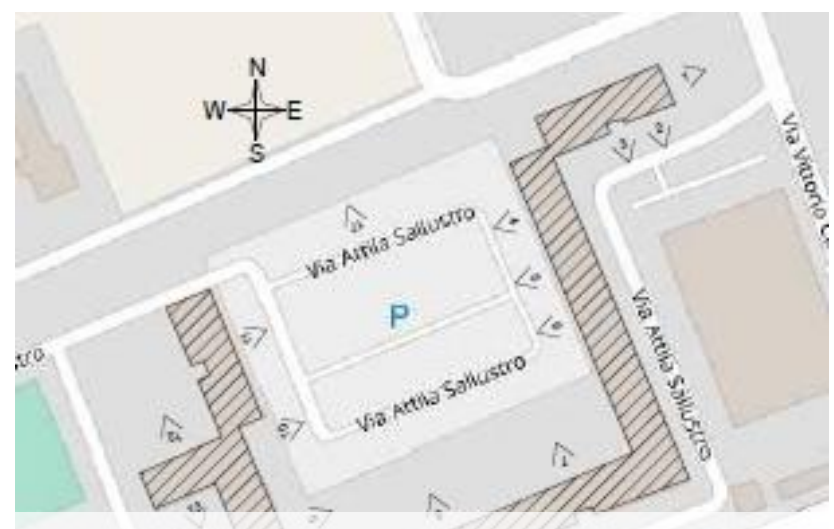

Figure 1. Distribution of the optical cones of the thermographic and photographic frames.

The photographic survey shows that the main typology of anomalies consist in crossshaped cracks in correst lines, in corresponden
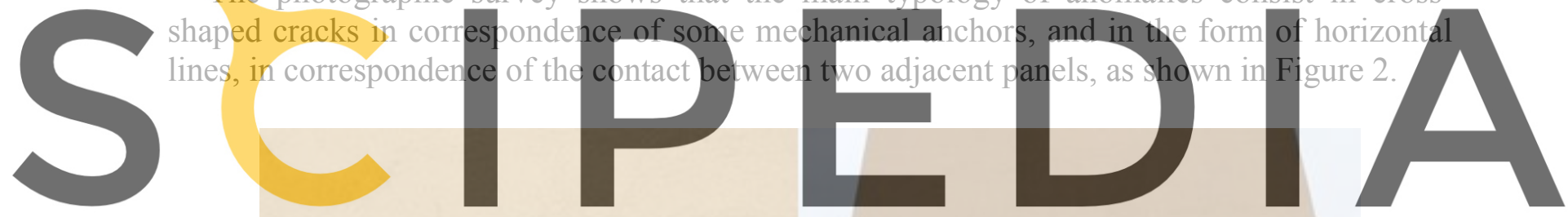

Register for free at https//www.scipedia.com to download the version without the watermark

a

Figure 2. Photographic frames: a) cross-shaped crack in correspondence of a mechanical anchor; b) line-shaped crack in correspondence of the contact between two adjacent panels.

Thermographic frames show color - corresponding to temperature - variations both in the shape of lines, which confirm the detachment revealed by the photographic information, and in the form of diffuse areas, as in Figure 3. 


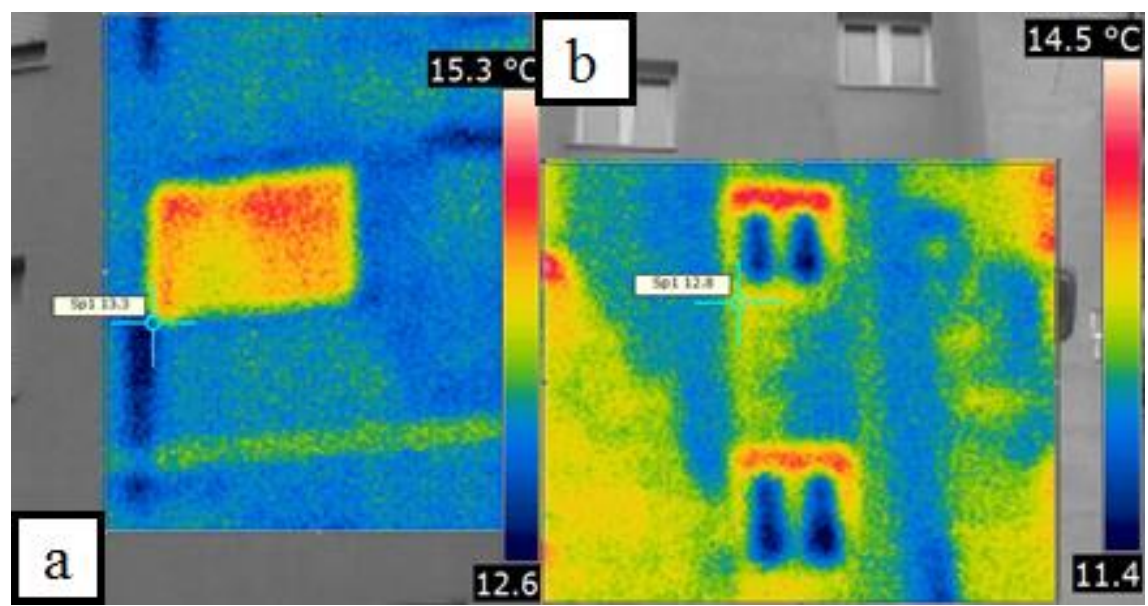

Figure 3. Thermographic frames: a) horizontal line at different temperature; b) diffuse thermal gradient on the surface.

\section{Results and Discussion}

A score between 1 and 5 was attributed to each of the photographic and thermographic frames, according to the typology and distribution of decay; the score of 5, corresponding to an unacceptable performance, was never attributed. Then, statistical analysis were performed

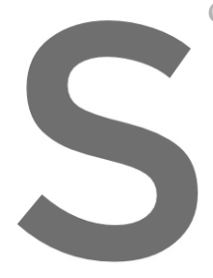
on the sample, leading
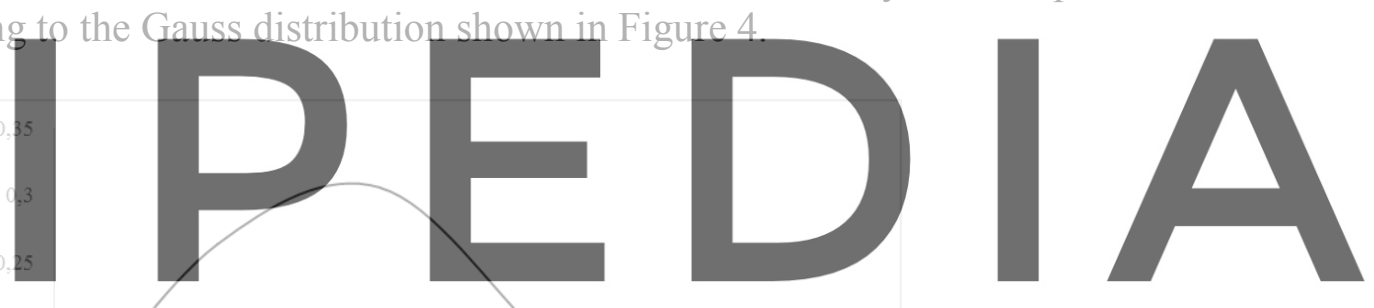

Register for free at https//www.scipedia.com to download the version without the watermark

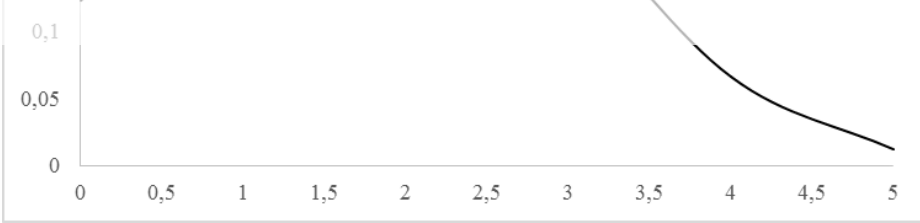

Figure 4. Gauss distribution of the state of decay of the ETICS in the experimentation, measured in the performance degrees of Table 1 .

The mean value of the performance decay is equal to 1.74 , while the standard deviation is 1.28. The result seems coherent with the age of the sample buildings, and is strongly dependent on the output of the thermographic survey. In fact, as it was partially pointed out in the previous paragraphs, several areas did not present any visible - nor sensible - decay in the external layers, but the thermographic frames showed a thermal gradient, which had to be related to a detachment of the panels, signalling a more consistent state of decay.

In order to break down the obtained data, it was chosen to perform also separate analyses by dividing the output according to the cardinal direction of the façades. This led to four 
different gauss distributions, respectively for the main cardinal directions of the building: ENE, N-NW, W-SW and S-SE, reported in Figure 5.

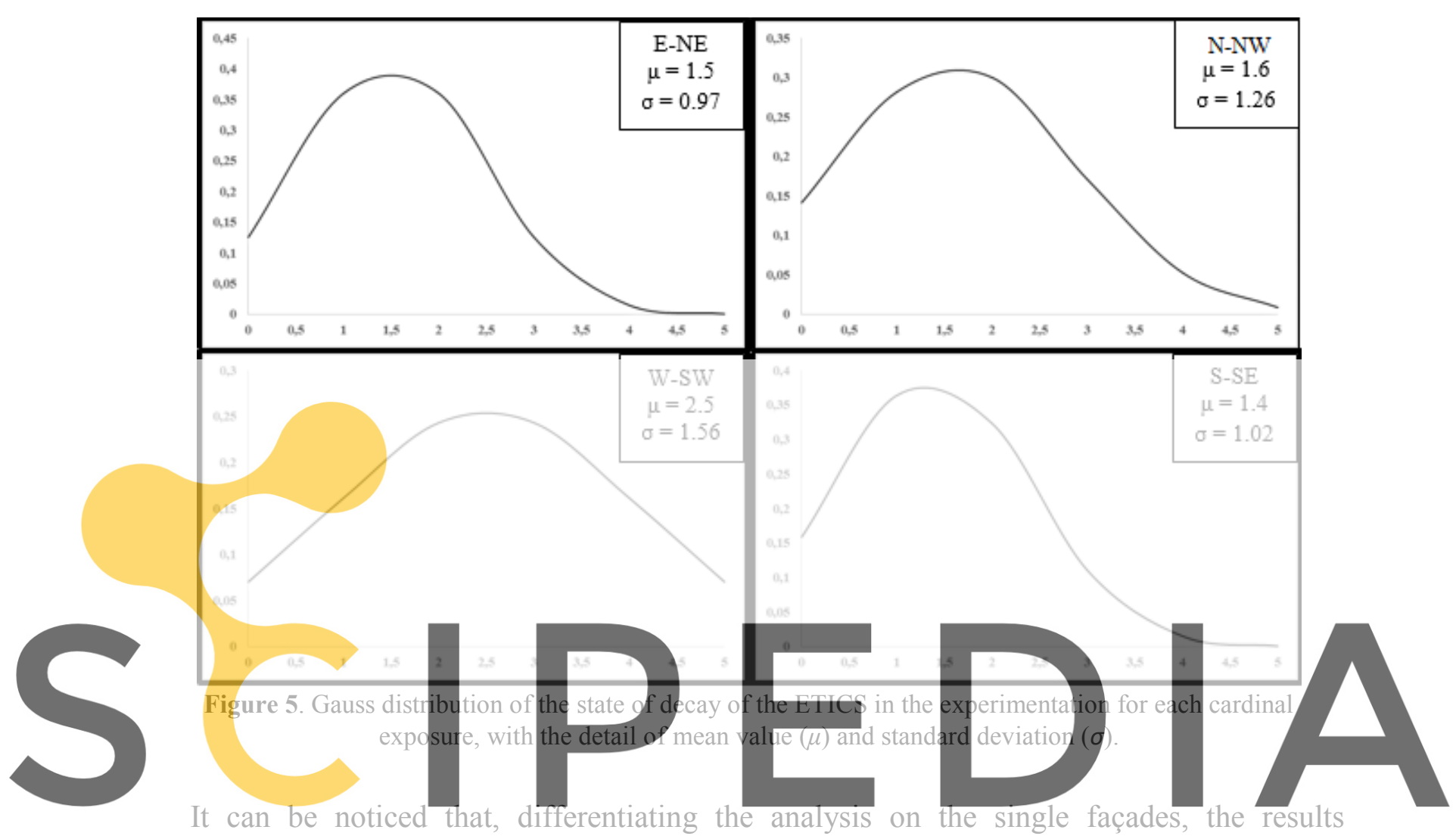

It can be noticed that, differentiating the analysis on the single facades, the results Register farfree at http more accurate: in 3 cases out of 4, the deviation is lower than in the the W-SW façade, while the other cardinal exposures have better performances. Since there is a higher frequency of detachment of panels, associated with higher performance degrees, this is also probably the reason behind the higher deviation.

The more negative value of performance decay in the W-SW direction seems particularly strong, reaching a ratio of 1.5-1.8 with the values in the other directions. Qualitatively, this phenomenon could be explained in relation to the less favorable conditions that insist on this façade, in particular solar exposure.

\section{Conclusions}

As for many materials in the sector of building constructions, the large use of ETICS in the current building industry does not find its counterpart in a sufficient quantity of field experimentations, compared to the notable number of theoretical models and laboratory tests; considering the complexity of the factors that influence this aspect in relation to the context, it is certainly necessary to encourage a more frequent use of field experimentations to enrich the database concerning the states of decay.

The aim of the experimentation carried out in this work was not to try to produce a relation 
between time and decay, as the collected data only refer to a single year, then it is not realistic to hypothesize a trend on its base, but rather the focus was on two significant aspects:

- the opportunities to make use of different tools and instruments for the monitoring activity, questioning their utility and the typology and level of information that they can provide;

- the qualitative differences that may exist in the decay of the same component, as a consequence of the variation of micro-contextual aspects.

The first point clearly refers to the use of the thermographic camera, and surely this case has provided a good proof of its peculiarities: incipient detachments between the panels could be noticed only thanks to the use of this instrument, allowing a much clearer picture of the state of decay; looking forward to the availability of quantitative data about the evolution of this anomaly, this is certainly an interesting information in the field of predictive maintenance.

At the same time, it has to be noticed that thermographic surveys do not show the dimension of the detachment, but only its existence; then, it is not possible to deduce the entity of the reduction of the thermal performance that the ETICS have to provide. So, an interesting development can be individuated in the possibility to link thermographic surveys to partially destructive tests, aimed to ascertain the dimension of the detachment, associate it to the output of the thermographic survey, and quantitatively determine its influence on the thermal performance of the ETICS.

Concerning the second aspect, the strong difference between the higher performance decay observed on one of the facades (W-SW) and the one on the others (E-NE, N-NW and S-SE) is certainly an interesting result, which is probably better not to
as the solar exposure might be, but rather to consider as an
perspective of more detailed assessments of the weight of
influence the durability of the single components. On this sid
will be interesting to gather data from thermographic surveys refer yet to a single parametor,
item of future interest, in the
the micro-climatic factors that
on for future experimentations, it geographic locations, in order to evaluate the entity of the differential performance degree

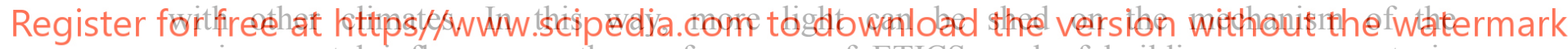
environmental influence on the performance of ETICS, and of buildings components in general.

\section{ORCID}

Maurizio Nicolella http://orcid.org/0000-0002-7140-6759

Roberto Landolfi http://orcid.org/0000-0002-5196-880X

Alessio Pino http://orcid.org/0000-0002-5774-6378

\section{References}

Bochen J., Gil S. and Szwabowski J. (2005). Influence of ageing process on porosity changes of the external plasters, Cement and Concrete Composites, 27, 769-775.

Daniotti B. and Paolini R. (2005). Durability Design of External Thermal Insulation Composite System with Rendering, Proceedings of the X International Conference on Durability of Building Components, Lyon, France.

Daniotti B. and Paolini R. (2008). Experimental programme to Assess ETICS cladding Durability, Proceedings of the XI International Conference on Durability of Building Materials and Components, Istanbul, Turkey.

Daniotti B., Re Cecconi F., Paolini R., Cocchetti G., Galliano R. and Cornaggia A. (2014). Multi-physics modelling for durability evaluation of ETICS, Proceedings of the XIII International Conference on Durability of Building Materials and Components, Sao Paulo, Brazil. 
Daniotti B., Spagnolo S. L. and Paolini R. (2008). Climatic Data Analysis to Define Accelerated Ageing for Reference Service Life Evaluation, Proceedings of the XI International Conference on Durability of Building Materials and Components, Istanbul, Turkey.

De Freitas S.S. and de Freitas V.P. (2016) Cracks on ETICS along thermal insulation joints: Case study and a pathology catalogue, Struct. Surv. 34, 57-72.

Minarovičová K. (2015). Environmental impact of ETICS Maintenance, Proceedings of the International Conference on Advanced Building Construction and Materials, Stražnice, Czech Republic.

Griciuté G., Bliūdžius R. and Norvaišienè R. (2013). The Durability Test Method for External Thermal Insulation Composite System (ETICS) used in Cold and Wet Climate Countries, Journal of Sustainable Architecture and Civil Engineering, 1, 50-56.

Koči V., Madera J. and Robert Č. R. (2012). Exterior thermal insulation systems for AAC building envelopes: Computational analysis aimed at increasing service life, Energy and Buildings, 47, 84-90.

Künzel H., Zirkelbach D. (2008). Influence of rain water leakage on the hygrothermal performance of exterior insulation systems, Proceedings of the 8th Symposium on Building Physics in the Nordic Countries, Copenhagen, Denmark.

Kvande T., Bakken N., Bergheim E. and Thue J.V. (2018). Durability of ETICS with Rendering in Norway Experimental and Field Investigations, Buildings 7, 1-17

Topcu D. and Merkel H. (2008). Durability of External Wall Insulation Systems with Extruded Polystyrene Insulation Boards, Proceedings of the XI International Conference on Durability of Building Materials and Components, Istanbul, Turkey.
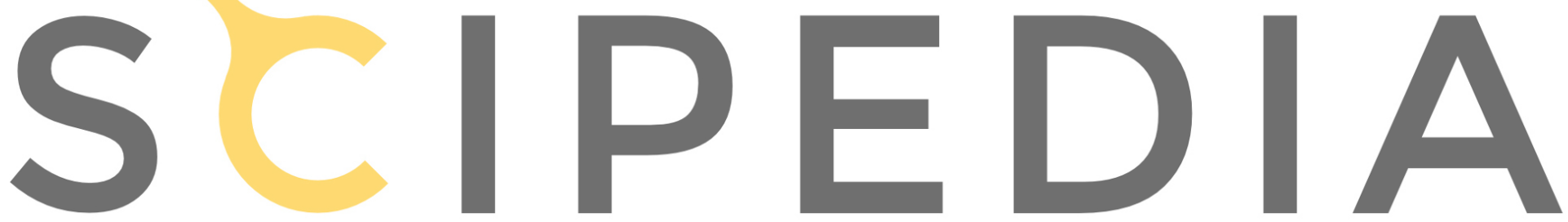

Register for free at https//www.scipedia.com to download the version without the watermark 